\title{
Spondylodesetechniken dorsal
}

Stefan Matschke, Klaus Wendl, Paul Alfred Gruetzner, Michael Kreinest, Sven Vetter

\section{Einleitung}

Differenzierte dorsale Instrumentierungen an der HWS ermöglichen die Versorgung unterschiedlicher Pathologien. Im Bereich der oberen HWS sind die dorsalen Instrumentierungen die am häufigsten zum Einsatz kommenden Operationstechniken.

Moderne Schrauben-Stab-Systeme ermöglichen eine sichere und komplikationsarme dorsale Stabilisierungsoption, sei es durch Schraubenplatzierung in der Massa lateralis oder in den Pedikeln. Dorsale Stabilisierungstechniken bieten dabei biomechanische Vorteile gegenüber einer ventralen Stabilisierung. Hauptindikation zur alleinigen dorsalen oder kombiniert dorsoventralen Spondylodese stellt die Versorgung von Verletzungen dar, einschließlich der Frakturen bei Spondylitis ankylosans. Die Stabilisierung von hochgradigen Instabilitäten bei Tumoren/Metastasen der HWS erfolgt in überwiegender Weise von dorsal und bietet gleichzeitig die Möglichkeit der Dekompression von dorsal. Sämtliche Spondylodesen im Übergangsbereich, ob okzipitozervikal oder zervikothorakal, erfolgen von dorsal.

Bedingt durch den unterschiedlichen anatomischen Aufbau der ersten beiden Halswirbel (Atlas und Axis) gegenüber C III bis C VII unterscheiden sich die dorsalen Stabilisierungstechniken der oberen HWS gegenüber der subaxialen HWS.

\section{Technik der dorsalen Stabilisierung der oberen HWS}

Als gute Verankerungsmöglichkeiten für Schrauben im Bereich der oberen HWS bietet sich die Schraube C I nach Harms ( $\mathbf{A b b} . \mathbf{8} \mathbf{e}, \mathbf{g} ; \mathbf{9} \mathbf{b}, \mathbf{c}$ ) [8] sowie die Pedikelschraube C II (Judet-Schraube; • Abb. 10 a, b) [10] an.

Ab C III bis C VI werden Schrauben in den Facetten als Massa-lateralis-Schrauben ( $\vee$ Abb. $\mathbf{5 f} ; \mathbf{6 c , d}$; $\mathbf{7 c , d}$; 8 c,d,f) [15] fixiert. In besonderen Situationen, bei denen kein ausreichender Schraubenhalt in den Facetten möglich ist, verwenden wir die Option der technisch aufwendigeren Pedikelschrauben ( $\bullet$ Abb. 9b, c). Ab C VII nach kaudal auf Th I, Th II ist das Einbringen der Pedikelschrauben anatomisch bedingt wieder vereinfacht möglich ( $\mathbf{A} \mathbf{b b} \mathbf{. 7} \mathbf{g}, \mathbf{h})$.
Für die C-I-Pedikelschraube bieten sich 2 Möglichkeiten der Schraubenplatzierung:

1. durch den hinteren Wirbelbogen in den Korpus C I $(\triangleright$ Abb. 5 c, d,e),

2. unterhalb des hinteren Wirbelbogens direkt in den Korpus C I.

Die erstere Technik erfordert anatomisch die Voraussetzung eines kräftigen hinteren Wirbelbogens, der die Schraube aufnehmen kann. Gleichzeitig bietet sie einen größeren Schraubenhalt.

\section{Merke}

Cave: Ein Abrutschen des Bohrers nach kranial oberhalb des hinteren Wirbelbogens birgt die potenzielle Gefahr der Verletzung der A. vertebralis.

Sind die Pedikel zu dünn für die Aufnahme einer Schraube, wird unterhalb des hinteren Wirbelbogens von lateral direkt auf den Korpus eingegangen.

\section{Merke \\ Cave: Unterhalb des hinteren Wirbelbogens muss die Nervenwurzel C I (sie enthält keine motorischen Fa- sern) sowie ein leicht verletzbarer venöser Plexus, der zu unübersichtlichen Blutungen im Verletzungs- fall führen kann, präparatorisch dargestellt werden.}

Die C-II-Pedikelschraube (Judet-Schraube, > Abb. 10 a, b) [10] hat für die Verankerungsmöglichkeit im Bereich der oberen HWS eine besondere Bedeutung, da hier durch den größten zu erreichenden Knochenkontakt ein besonders guter Schraubenhalt erzielt werden kann. Es lassen sich i.d.R. Schrauben mit einem Durchmesser von 4 mm platzieren.

Nach Josten [4] bietet die C-II-Pedikelschraube eine 2fach höhere Ausrissfestigkeit im Vergleich zu Schrauben, die in der HWS-Facette eingebracht werden.

Gleichzeitig kann sie durch eine ansteigende Bohrrichtung transfixierend über die Gelenke C I/C II eingebracht werden (Magerl-Technik) [9,23] und bietet damit eine noch höhere Stabilität. ( $\bullet$ Abb. 1 d, e,f; 2; 3 c; 4 h, i).

Die heute zur Verfügung stehenden polyaxialen Schraubensysteme mit den differenzierten Angulationen der 

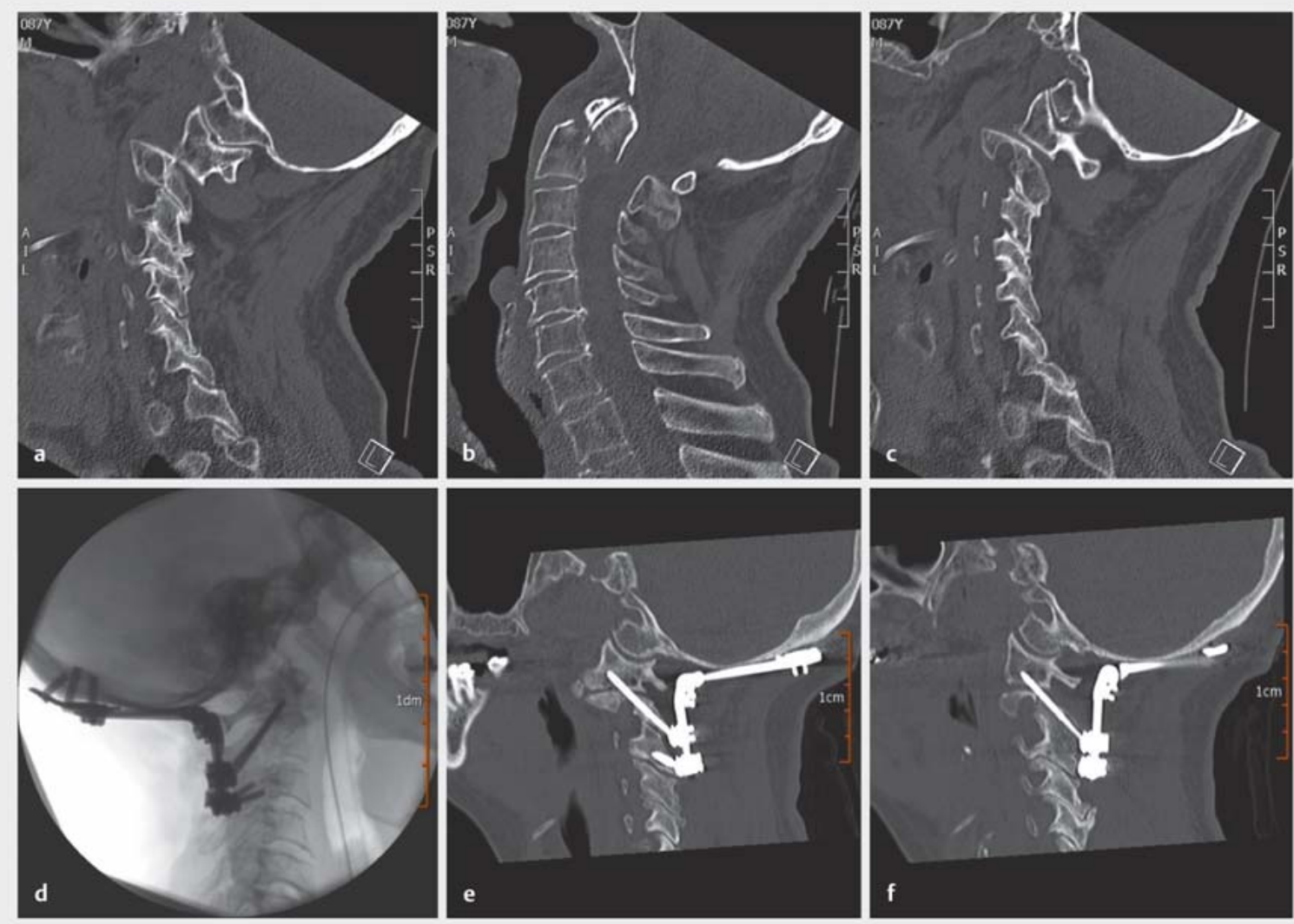

- Abb. 1 Dislozierte Densfraktur mit Luxation der Gelenke C I/C II bei translatorischer atlantoaxialer Instabilität (a,c). d Intraoperatives Durchleuchtungsbild nach Reposition der Densfraktur sowie der Gelenke C I/C II, Stabilisation über okzipitozervikale Instrumentierung C 0 auf C III mit Transfixation der Gelenke C I/C II nach Magerl. e und f Sagittale CT-Rekonstruktionen in Höhe der Gelenke C I/C II nach Reposition und Transfixation der Gelenke C I/C II nach Magerl.

Schraubenköpfe sowie die Möglichkeit des risikoarmen Einbringens der C-I- und C-II-Pedikelschrauben unter Anwendung von intraoperativen 3-D-Bildwandlerkontrollen und der Navigation haben zu einem Wechsel der früher etablierten dorsalen Operationstechniken unter Verwendung von Cerclagen, wie der OP nach Gallie [6], zugunsten der biomechanisch stabileren Schrauben-Stab-Instrumentierungen geführt.

Als Beispiel hierfür zu nennen ist die C-I-C-II-SchraubenStab-Instrumentierung nach Harms-Goehl ( $\boldsymbol{A} \mathbf{A b b} . \mathbf{3 c}$ ) [8].

Alternativ ist die Kombination eines Schrauben-Stab-Systems mit Fixierung über Atlashaken möglich. Voraussetzung ist die Integrität des hinteren Atlasrings. Durch Transfixierung der Gelenke C I/C II im Sinne einer Magerl-Verschraubung $[9,23]$ lässt sich eine biomechanisch äußerst stabile C-I-C-II-Fixierung erreichen ( $\bullet$ Abb. 2).
Wenn immer möglich, gilt es, im Bereich der oberen HWS die Funktion $\mathrm{Co} / \mathrm{C}$ I zu erhalten. Die Kopffunktionen mit der Möglichkeit der Reklination wie auch Inklination sind in der Ausübung der Aktivitäten des täglichen Lebens (ADL), wie bspw. auch Essen und Trinken, von enormer Bedeutung. Eine ausreichende Kompensationsmöglichkeit besteht, wenn noch keine übermäßigen degenerativen Veränderungen der subaxialen HWS vorliegen.

Der Hautschnitt für das Einbringen der C-I- bzw. C-II-Pedikelschrauben beginnt unterhalb der Protuberantia occipitalis externa und zieht in der Medianlinie nach kaudal. Für den Eintrittspunkt der C-II-Pedikelschraube wird der Isthmus subperiostal freipräpariert. Der Eintrittspunkt für die Schraube befindet sich zwischen Lamina und Gelenkfortsatz in der Mitte des Isthmus. Gebohrt wird unter seitlicher Bildwandlerkontrolle mit Zielrichtung auf den vorderen Atlasbogen. 


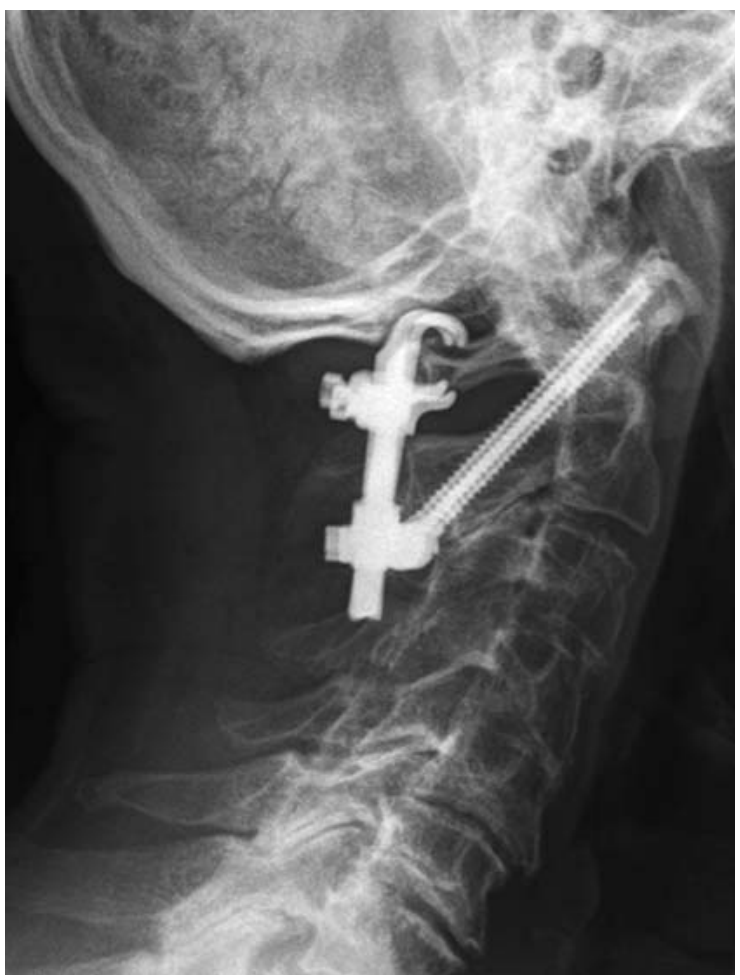

Abb. 2 Atlantoaxiale Instrumentierung monosegmental über Atlashaken in Kombination mit einer transartikulären Verschraubung C I/C II nach Magerl bei Densfraktur mit translatorischer atlantoaxialer Instabilität.

Cave

Da die Schraubenrichtung am Oberrand des Foramens $\operatorname{der} A$. vertebralis verläuft, muss zuvor eine „highriding-A.-vertebralis“ durch Analyse eines präopera- tiven CT ausgeschlossen werden bzw. die Schraubenrichtung genau geplant werden ( $\bullet$ Abb. $1 \mathbf{e}, \mathbf{f})$.

Beachte Schraubenrichtung bei „high-riding-A.-vertebralis" am Oberrand des Canalis der A. vertebralis ( Abb. 1 b).

Für das Einbringen der C-I- und C-II-Pedikelschraube ist eine intraoperative 3-D-Bildgebung oder die Anwendungsmöglichkeit der Navigation von großem Vorteil $[17,19]$.

\section{Technik der dorsalen Stabilisierung der subaxialen HWS}

Für die Schraubenplatzierung der subaxialen HWS sind zahlreiche Techniken beschrieben [7]. Die am häufigsten und am einfachsten zu verwendende Technik ist die Besetzung der Schrauben in den Facetten als Massa-lateralis-Schraube ( $\bullet$ Abb. 5f; $\mathbf{6 c}$, d; 7 c, d; 8 c, d,f). Eine weitere, technisch deutlich anspruchsvollere Schraubenpositionierung im Bereich der subaxialen HWS ist die Verwendung von Pedikelschrauben ( $\boldsymbol{A}$ Abb. $\mathbf{9 b}, \mathbf{c}$ ). Im Vergleich beider Techniken bieten die Pedikelschrauben den klaren biomechanischen Vorteil einer erhöhten Stabilität und Ausreißfestigkeit.

Beide Techniken nutzen die modernen Schrauben-StabSysteme mit Polyaxialschrauben [14].

Analog zur Platzierung der Schrauben im Bereich der oberen HWS liegt der Patient in Bauchlage und der Hautschnitt verläuft in der Medianlinie über den Dornfortsätzen nach kaudal. Die paravertebrale Muskulatur wird idealerweise subperiostal unter sorgfältiger Blutstillung
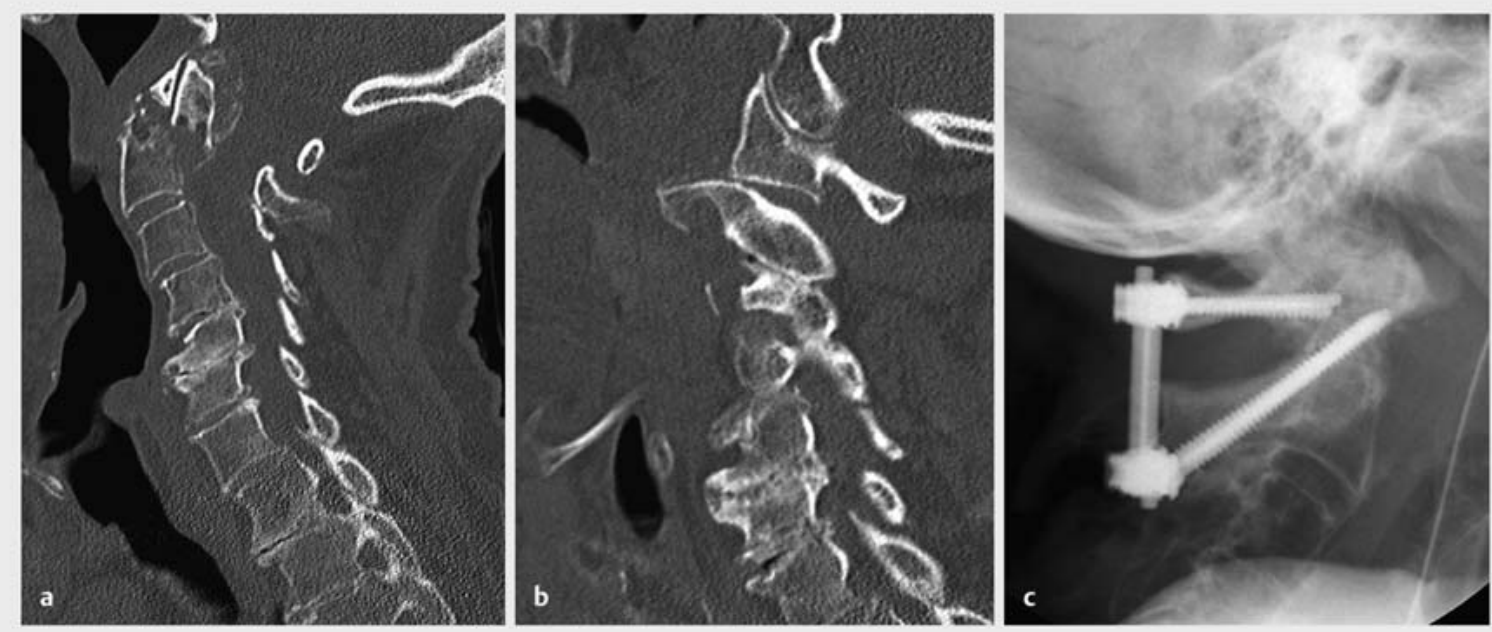

- Abb. 3 a, b Dislozierte Densfraktur bei translatorischer atlantoaxialer Instabilität, Luxation der Gelenke C I/C II. c Dorsale atlantoaxiale Instrumentierung nach Harms-Goehl. 

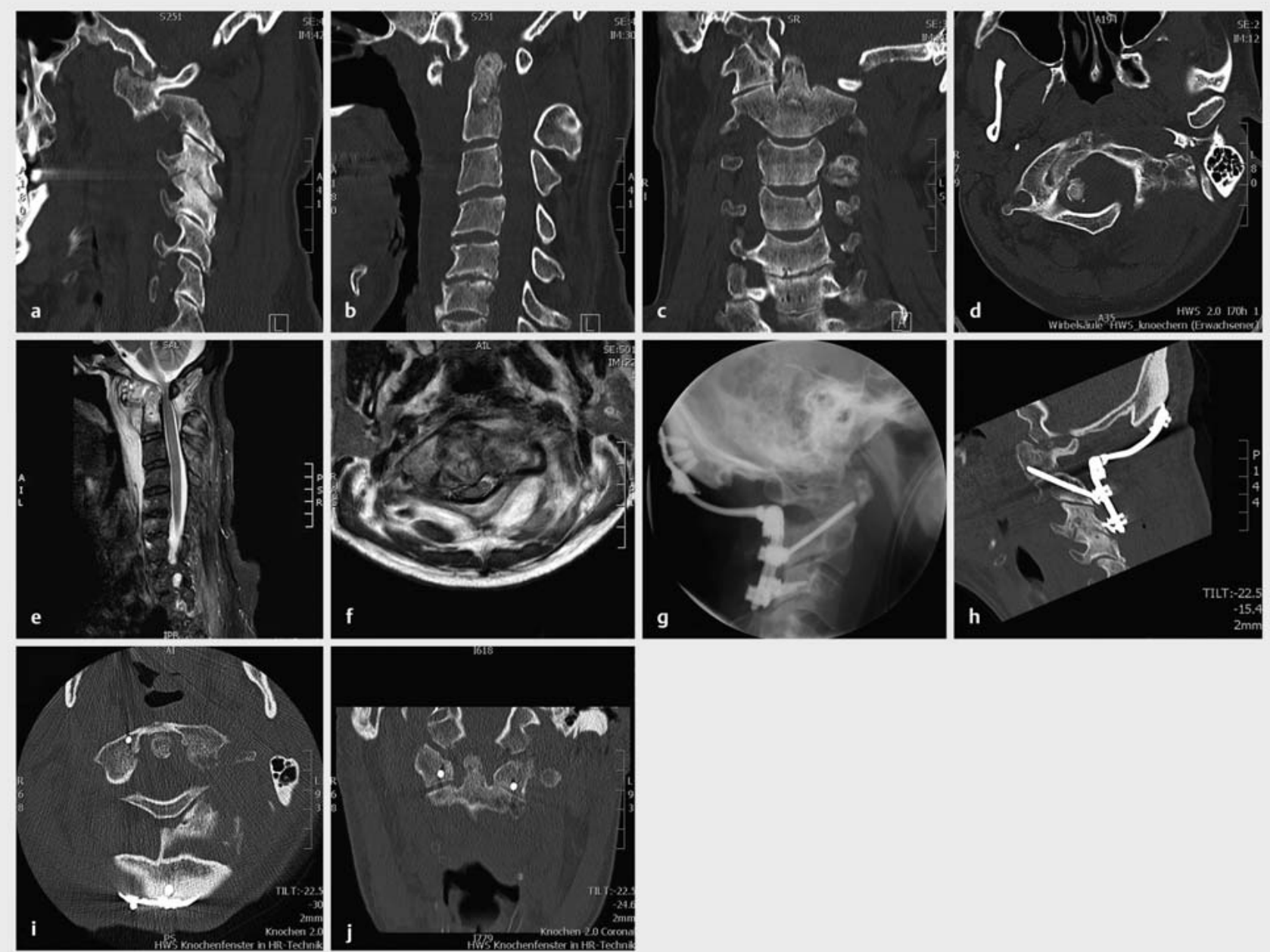

- Abb. 4 a-c Anteriore translatorische Fehlstellung C I/C II Fielding Grad 3 mit einseitiger Luxation der Gelenke C I/C II und begleitender Densfraktur. d Axiales Schnittbild in Höhe C I mit rotatorisch dislozierter Densspitze nach lateral und Rotation des Axis nach anterior. e, $\mathbf{f}$ Sagittale und axiale Schnittbildgebung MRT mit Darstellung der hochgradigen Spinalkanalstenose durch die rotatorisch-anteriore Fehlstellung des Dens axis. g, h Okzipitozervikale Instrumentierung C0 auf C III, sagittale CT-Rekonstruktionen in Höhe der Gelenke C I/C II nach Reposition und Transfixation der Gelenke C I/C II nach Magerl. i Axiale CT-Rekonstruktion in Höhe C I mit regelrechter Stellung des Dens axis. j Koronare CT-Rekonstruktion in Höhe der Gelenke C I/C II.

von den Dornfortsätzen präpariert, bis die Wirbelbögen erreicht sind.

Die Facetten zur Aufnahme der Massa-lateralis-Schrauben werden von den Wirbelbögen nach lateral unter weiterer Ablösung der paravertebralen Muskulatur dargestellt. Um die Massa-lateralis-Schrauben korrekt zu positionieren, ist die anatomische Orientierung mit der kraniokaudalen wie auch der mediolateralen Ausdehnung der Facette erforderlich [15].

Während Magerl [9] den idealen Eintrittspunkt ca. 1$2 \mathrm{~mm}$ medial und kranial der Mitte der Massa lateralis sieht und die Bohrrichtung ca. 30-40 nach kranial und ca. $25^{\circ}$ nach lateral, möglichst parallel zur Gelenkfläche beschreibt, liegt in der Beschreibung nach Roy-Camille
[10] der Eintrittspunkt in der Mitte der Massa lateralis. Die Bohrrichtung ist in seiner Beschreibung anterior-posterior, rechtwinklig zur Massa lateralis um ca. $10^{\circ}$ nach lateral geneigt.

Einen evidenzbasierten Vorteil einer dieser verschiedenen Techniken gibt es nicht. Exzellente Fusionsraten bis 99\% unter Verwendung von Massaschrauben sind beschrieben [23].

Die technisch erheblich anspruchsvollere Pedikelschraube kann bei hochgradigen Instabilitäten bei schlechten Knochenverhältnissen, wie bei älteren Patienten mit Osteoporose oder Patienten mit Morbus Bechterew, mit hohen Festigkeitsansprüchen bei großen Hebelverhältnissen von erheblichem Vorteil sein [5]. 

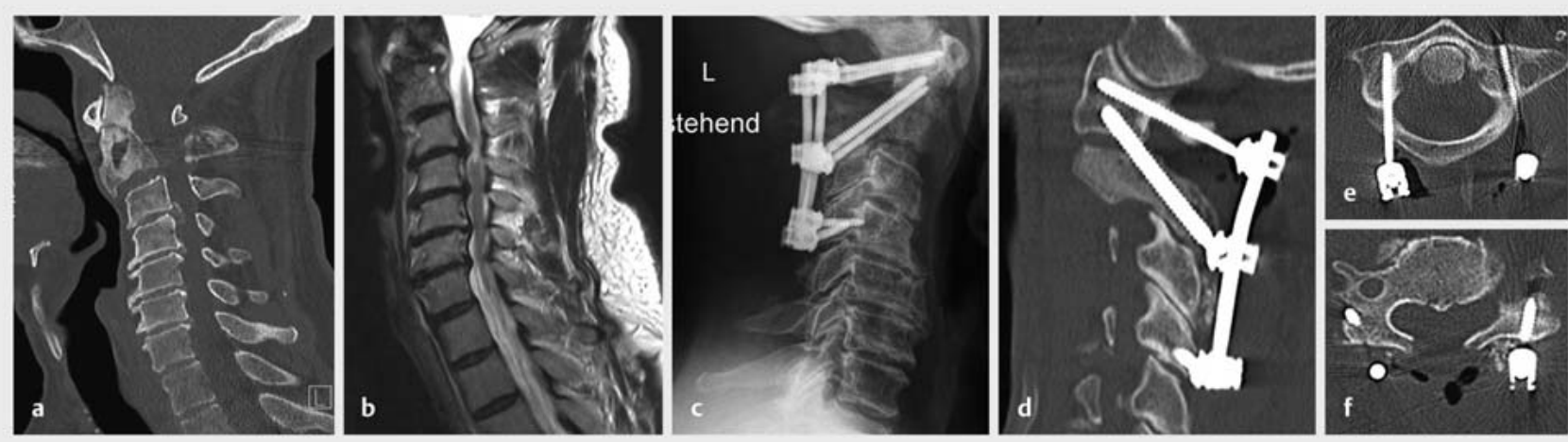

- Abb. 5 a, b Myelonkontusion C III/C IV bei Spinalkanalstenose nach Hyperflexionstrauma, Densfraktur Typ Anderson II. c, d Dorsale Instrumentierung C I/C II (Transfixation C I/C II nach Magerl) auf C IV (Massa-lateralis-Schrauben) mit Dekompression durch Laminektomie C III/C IV, Anlagerung von Actifuse ${ }^{\circledR}$ zur dorsalen Fusion. e, $f$ Schraubenlagen axial C I und C IV mit Dekompression durch Laminektomie C III/C IV.
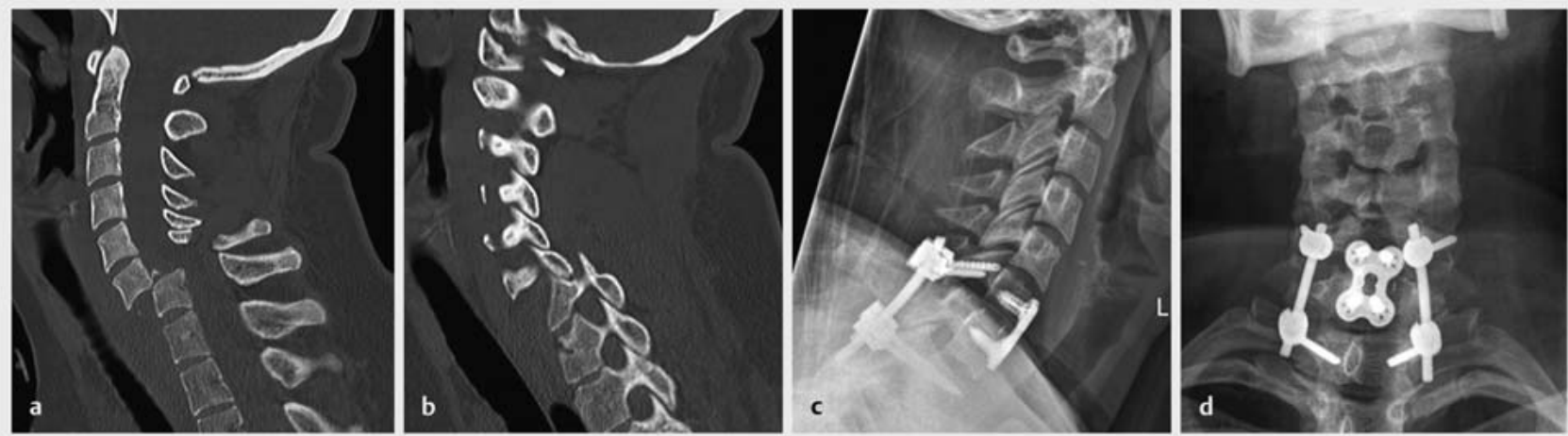

- Abb. 6 a, b C VI/VII-Luxationsverletzung mit beidseitiger verhakter Luxation der kleinen Wirbelgelenke, (AO Spine: C6/7; C; F4, BL; N4). c, d C VI/VII dorsoventrale Spondylodese; dorsale Reposition und Instrumentierung von C VI auf Th I (C VI-Massa-lateralis-Schrauben; Th-IPedikelschrauben), ventrale monosegmentale Fusion C VI/VII.

Um die Eintrittspunkte von Pedikelschrauben der subaxialen HWS darzustellen, ist die Verwendung von lateralen Zusatzinzisionen erforderlich. Die erforderliche Konvergenz dieser Pedikelschrauben von annähernd $30^{\circ}$ und der extrem laterale Eintrittspunkt lässt sich über eine mediane Schnittführung nur sehr aufwendig bis gar nicht erreichen.

Die Verwendung von Pedikelschrauben im Bereich der subaxialen HWS erfordert den Einsatz der Navigation $[17,19]$ bzw. einer intraoperativen 3-D-Bildgebung. Idealerweise werden nach Präparation des typischen Eintrittspunktes der Pedikelschraube, die sich nach Abumi et al. $[1,2]$ in Höhe C III bis C VII leicht seitlich des Zentrums des Gelenkfortsatzes nahe am Unterrand des inferioren Gelenkfortsatzes des darüber liegenden Wirbels befindet, Drähte über die Pedikel in den Korpus vorgebohrt. Zur Orientierung der kraniokaudalen Ausrichtung orien- tiert man sich am intraoperativen Seitbild. Nach Überprüfung der korrekten Lage der Drähte und Schneiden eines Gewindes werden die Pedikelschrauben eingedreht.

Nur sehr wenige Implantathersteller bieten gegenwärtig kanülierte Systeme für eine Pedikelschraubenpositionierung an der HWS an (Neon III ${ }^{\circledR}$.

\section{Merke}

Cave: Anhand des präoperativen CT muss die Pedikelbreite bestimmt werden. Bei einem Pedikeldurchmesser unter $3 \mathrm{~mm}$ sollte auf die Verwendung von Pedikelschrauben verzichtet werden. Es resultieren sonst beim Einbringen der Pedikelschraube zwangsläufig Verletzungen des Spinalkanals bzw. des Foramens der A. vertebralis mit der Gefahr von neurovaskulären Schäden. 

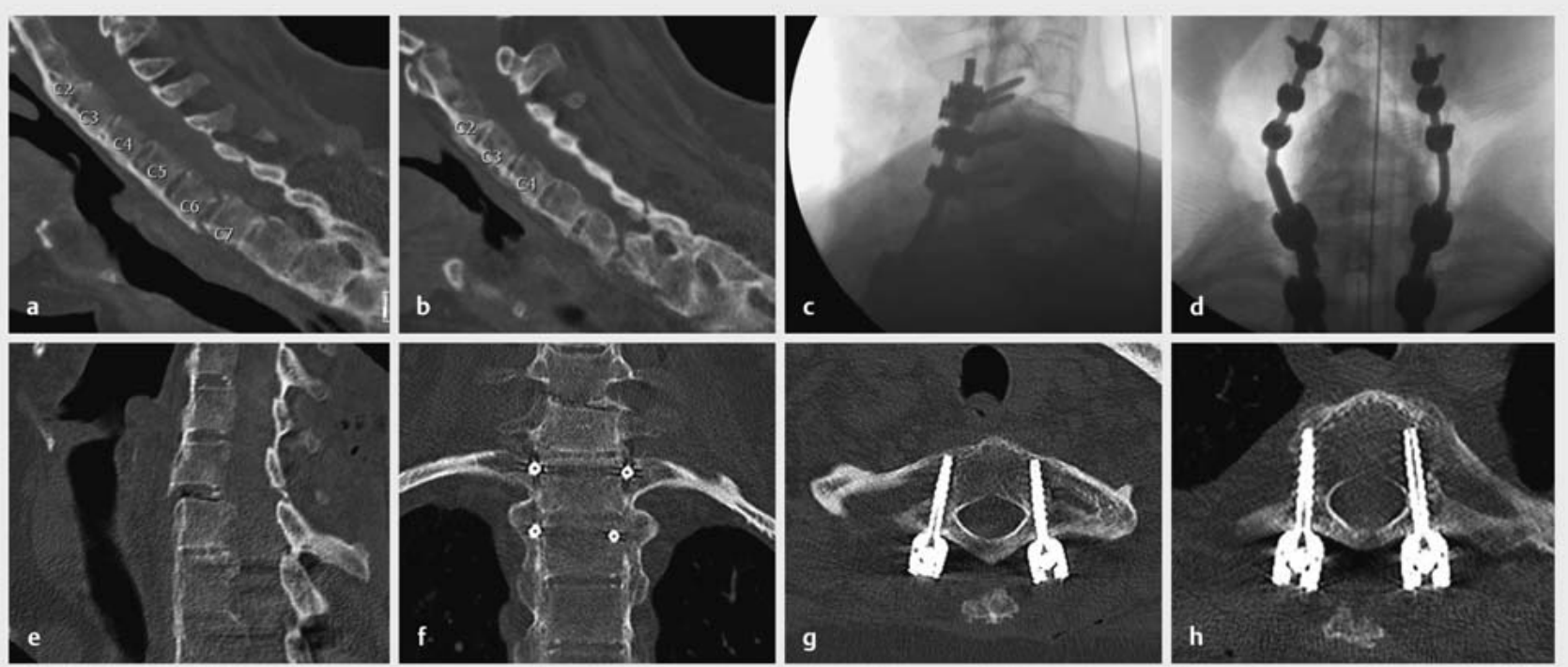

- Abb. 7 a, b Ventrale und dorsale Zerreißung bei Morbus Bechterew im Segment C VI/C VII (AO Spine: C6/7; C, N0, M3). c, d Langstreckige dorsale Instrumentierung C IV, C V, C VI (Massa-lateralis-Schrauben) auf Th I, Th II (Pedikelschrauben). e, f Sagittale und koronare Rekonstruktionen, postoperatives CT. g, h Axiale Schnittbilder Th I, Th II, Pedikelschrauben nach perkutaner Instrumentierung.

Auch wenn für die Positionierung von Pedikelschrauben im Vergleich zu Massa-lateralis-Schrauben ein theoretisch deutlich erhöhtes Komplikationsrisiko besteht, ist jedoch die Komplikationsrate in der Hand des Erfahrenen erstaunlich gering [22].

Um den präparatorischen Aufwand zu minimieren, sind hier auch minimalinvasive Techniken sowohl für die Platzierung von Massa- als auch von Pedikelschrauben $(\bullet \mathbf{A b b} . \mathbf{~ g}, \mathbf{h})$ beschrieben $[11,13]$.

\section{Indikationen zur dorsalen}

\section{Stabilisierung der oberen HWS}

Bis auf die ventrale Schraubenosteosynthese des Dens axis, der ventralen Verschraubung der Gelenke C I/C II nach Barbour [3], besteht die Indikation für ein ventrales Vorgehen vorwiegend bei Instabilitäten, die das Segment C II/C III betreffen.

Hier sind es die Verletzungsfälle der traumatischen Spondylolisthese mit Verletzung des vorderen Längsbandes, bei denen noch keine vollständige Luxation der kleinen Wirbelgelenke vorliegt (Typ Josten 3) [4].

Die überwiegende Anzahl aller Instabilitäten und Verletzungen der oberen HWS, die einer operativen Therapie zugeführt werden müssen, werden ausschließlich oder primär von dorsal stabilisiert:
1. instabile Atlasfrakturen Typ Gehweiler 3b (=kombinierte vordere und hintere Atlasbogenfraktur mit Zerreißung des Lig. transversum)

2. Densfrakturen mit begleitender atlantoaxialer Instabilität ( Abb. 1 a-c; $>\mathbf{3 a}$ a, b) [12]

3. traumatische Spondylolisthese C II/C III Typ Josten 4 ( Abb. 9a)

4. ligamentäre Verletzungen der oberen Halswirbelsäule (okzipitozervikale Dislokation CO/C I; axiale atlantoaxiale Instabilität C I/C II; translatorische atlantoaxiale Instabilität C I/C II; rotatorische atlantoaxiale Instabilität C I/C II ( $\mathbf{A b b}$. 4-g, j, k)

5. kombinierte traumatische Instabilitäten mit degenerativen Stenosen ( $\bullet \mathbf{A b b} \mathbf{5} \mathbf{a}, \mathbf{b})$

6. Instabilitäten bei Tumoren/Metastasen der oberen HWS

7. Operationsindikationen aufgrund einer rheumatischen Erkrankung

\section{Indikationen zur dorsalen}

\section{Stabilisierung der subaxialen HWS}

1. Dorsale ligamentäre/knöcherne Instabilität (Frakturen vom Typ B und C mit luxierten kleinen Wirbelgelenken) in Kombination mit ventraler Fusion ( $\bullet$ Abb. $\mathbf{6} \mathbf{a}, \mathbf{b}$ )

2. Frakturen bei ankylosierender Spondylitis $(\triangleright$ Abb. 7 a, b,e,f)

3. Instabilitäten bei Tumoren/Metastasen der subaxialen HWS ( $\bullet$ Abb. 8 a, b)

4. Instabilitäten und Verletzungen im zervikothorakalen Übergangsbereich 

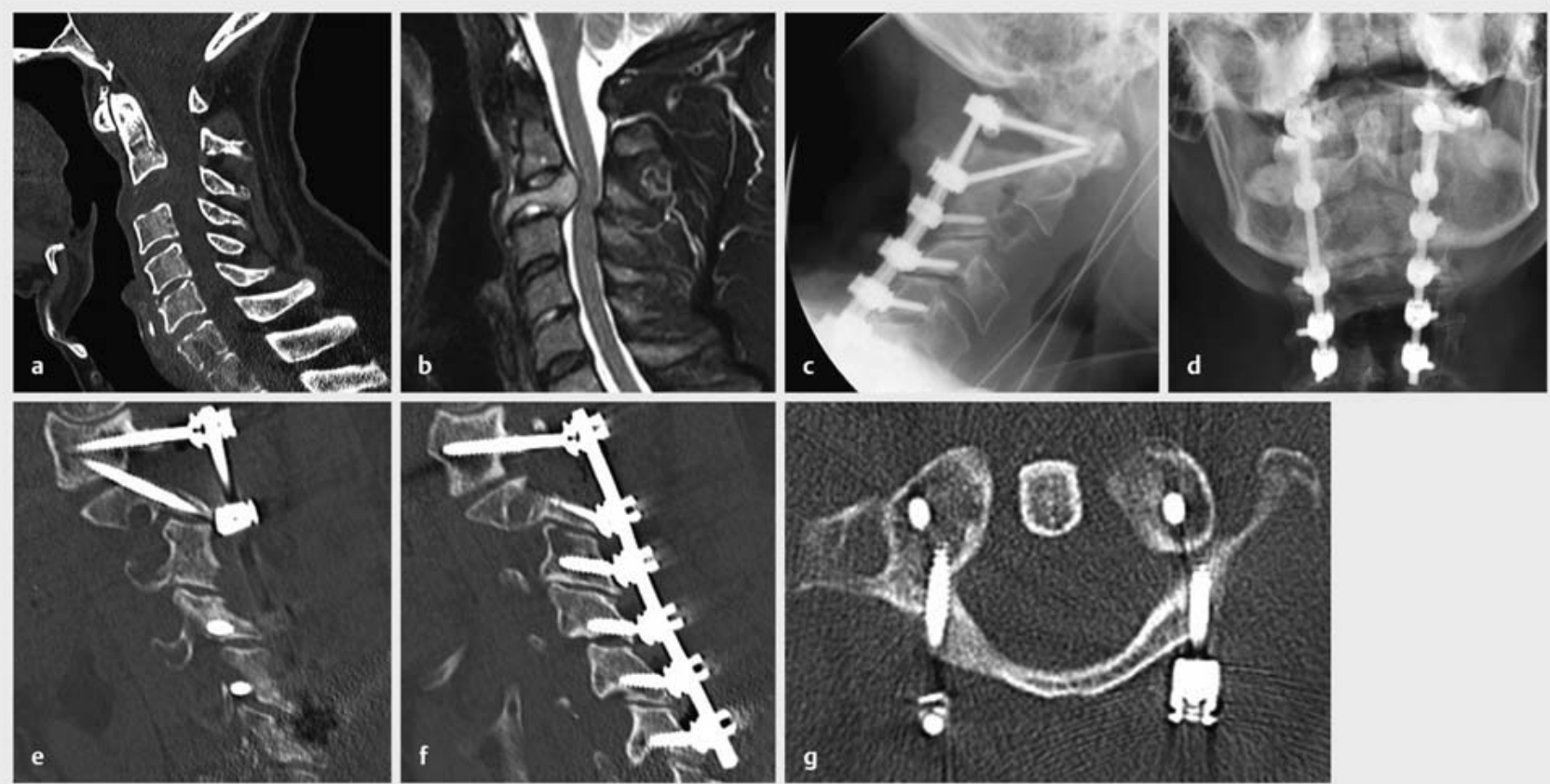

- Abb. 8 a, b CT und MRT HWS bei multiplem Myelom mit diffuser Metastasierung, hochgradige Instabilität bei Destruktion C III mit Spinalkanalstenose. c, d Langstreckige dorsale Instrumentierung von C I, C II (nach Harms-Goehl) auf C III (nur links), C IV, C V, C VI (beidseits) mit Massalateralis-Schrauben, Dekompression C III durch Laminektomie. e, f Schraubenlagen C I, C II, C III, C IV, C V, C VI sagittale CT-Rekonstruktionen. g Schraubenlagen $\mathrm{Cl}$ axial.
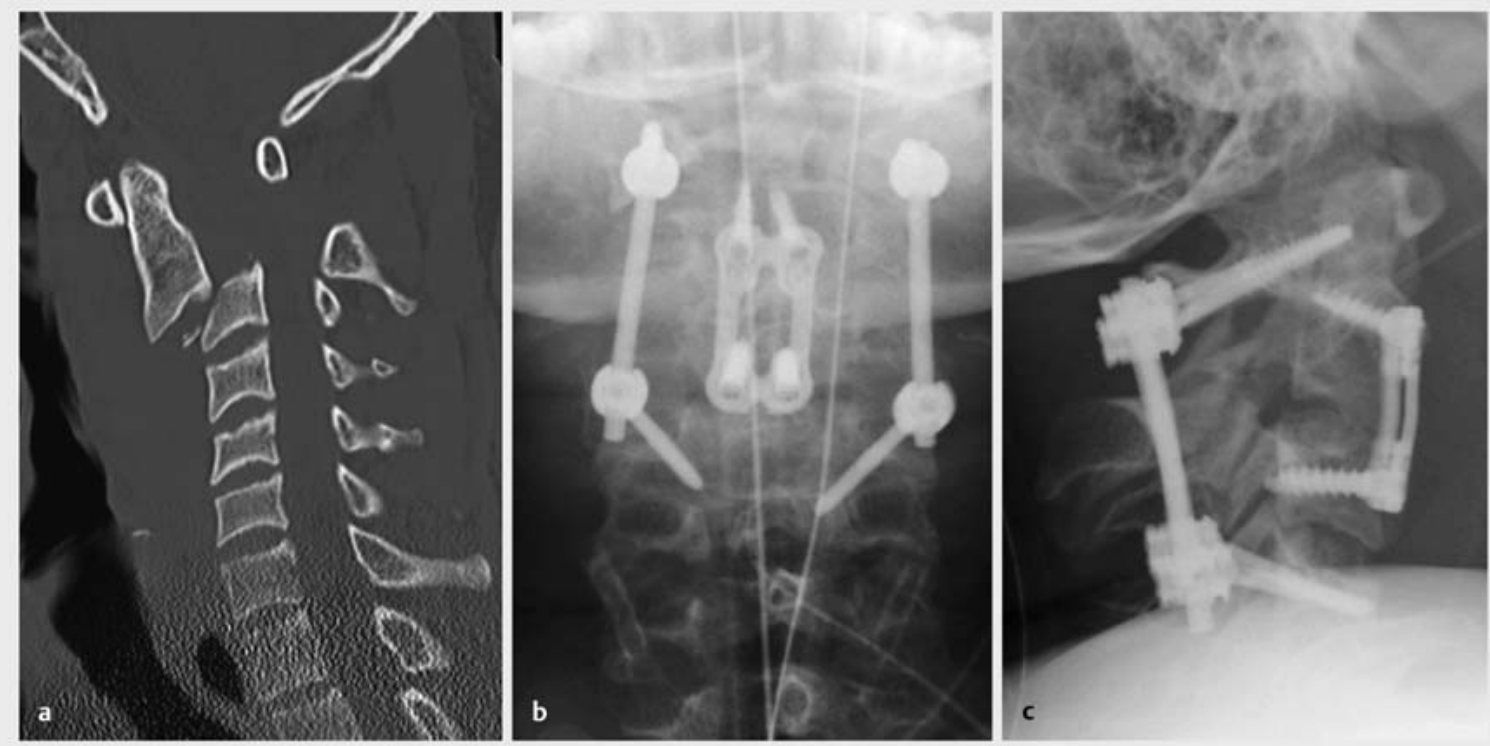

- Abb. 9 a Traumatische Spondylolisthese C II/C III mit Ruptur ALL (ALL: vorderes Längsband), Bandscheibenzerreißung C II/C III, Luxation der Gelenke C II/C III Typ Josten 4. b Frontales postoperatives Bild nach einzeitig dorsoventraler Spondylodese mit Pedikelschrauben-Stab-System C I und C III, ventral monosegmentale Spondylodese C II/C III. C Sagittales postoperatives Bild nach einzeitig dorsoventraler Spondylodese mit Pedikelschrauben-Stab-System C I auf C III, ventral monosegmentaler Fusion C II/C III. 
Das ventrale operative Zugehen in vorgegebenen anatomischen Schichten und die einfache Darstellungsmöglichkeit der subaxialen HWS von vorn ist einer der wesentlichen Gründe dafür, dass im Verletzungsfall die überwiegende Anzahl aller Verletzungen der subaxialen HWS von ventral reponiert und fusioniert wird $[16,18]$.

Trotz alledem gilt es, im Falle einer traumatisch bedingten Instabilität im subaxialen Bereich diese Verletzungen zu detektieren, bei denen primär das dorsale Vorgehen indiziert ist [14]. Hier handelt es sich um Verletzungen vom Typ C oder B mit Luxationen der kleinen Wirbelgelenke, bei denen eine ventrale Reposition nicht möglich ist oder von dorsal primär eine Dekompression erforderlich wird ( $\triangleright$ Abb. 6a, b).

Bei den hochgradig instabilen C-Verletzungen kann die alleinige ventrale Fusion nach Reposition die biomechanisch erforderliche dorsale Zuggurtung nicht wiederherstellen und ein additives dorsales Vorgehen ist hier indiziert als ventrodorsales Verfahren oder primär als dorsoventrales Verfahren $[18,20]$.

\section{Fazit}

Die dorsale Spondylodese bzw. die dorsale Instrumentierung ist integraler Bestandteil bei der Versorgung von unterschiedlichen Wirbelsäulenpathologien der HWS. Die dorsale Spondylodese ist die Domäne bei der Versorgung von Verletzungen im Bereich der oberen HWS mit der Möglichkeit unterschiedlicher Operationsverfahren. Am häufigsten hierbei kommen die Pedikelschrauben in C I und/oder C II zum Einsatz.

Während bei subaxialen Verletzungen die überwiegende Anzahl initial von ventral versorgt wird, stellen gerade die hoch instabilen Verletzungen vom Typ C [21] mit luxierten Gelenken auch im Bereich der subaxialen HWS eine Indikation für ein kombiniertes dorsoventrales Verfahren dar. Durch das technisch einfache Besetzen von Massalateralis-Schrauben in den Facetten ist diese Schraubenbesetzung für die allermeisten Fälle der subaxialen HWS ausreichend. Biomechanisch bieten die technisch aufwendigeren Pedikelschrauben im Bereich der subaxialen HWS eine gute Alternative bei langen Hebelwirkungen bspw. bei Osteoporose bei Patienten mit Morbus Bechterew. Standardverfahren sind Massaschrauben und Pedikelschrauben, in der Hand des Erfahrenen treten dabei keine gehäuften Komplikationsraten auf.

Interessenkonflikt

Die Autoren geben an, dass kein Interessenkonflikt besteht.

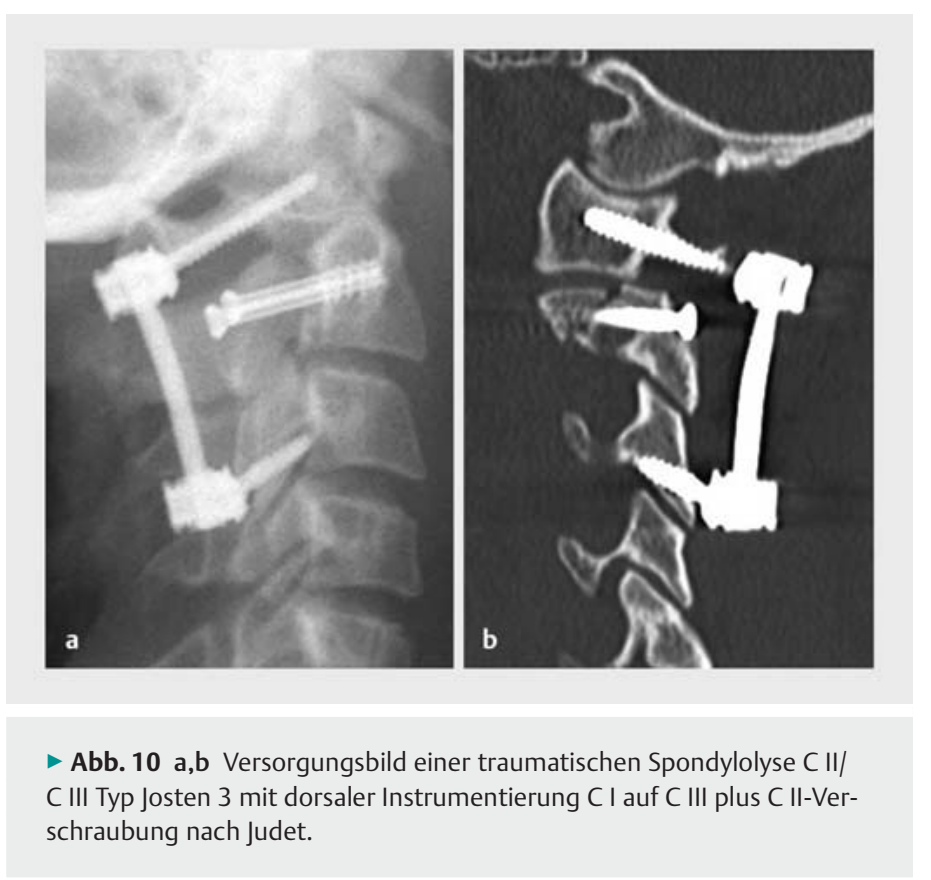

Autorinnen/Autoren

\section{Stefan Matschke}

Dr. med., Leiter Sektion Wirbelsäulenchirurgie, Klinik für Orthopädie und Unfallchirurgie, BG Klinik Ludwigshafen

\section{Klaus Wendl}

Dr. med., Klinik für Orthopädie und Unfallchirurgie, BG Klinik Ludwigshafen

\section{Paul Alfred Gruetzner}

Prof. Dr. med., Klinik für Orthopädie und Unfallchirurgie, BG Klinik Ludwigshafen

\section{Michael Kreinest}

Dr. Dr., Klinik für Orthopädie und Unfallchirurgie, BG Klinik Ludwigshafen

\section{Sven Vetter}

Dr., Klinik für Orthopädie und Unfallchirurgie, BG Klinik Ludwigshafen

\section{Korrespondenzadresse}

\author{
Dr. med. Stefan Matschke \\ Die Wirbelsäule \\ Zentrum für Wirbelsäulenchirurgie an der \\ ATOS Klinik Heidelberg \\ Bismarckstraße 9-15 \\ 69115 Heidelberg \\ stefan.matschke@atos.de
}




\section{Literatur}

[1] Abumi K, Ito M, Sudo H. Reconstruction of the subaxial cervical spine using pedicle screw instrumentation. Spine (Phila Pa 1976) 2012; 37: E349-E356

[2] Abumi K, Shono $Y$, Kotani $Y$ et al. Indirect posterior reduction and fusion of the traumatic herniated disc by using a cervical pedicle screw system. J Neurosurg 2000; 92: 30-37

[3] Barbour JR. Screw fixation in fractures of the odontoid process. S Aust Clinics 1971; 5: 20

[4] Bühren V, Josten C. Chirurgie der verletzten Wirbelsäule. Berlin, Heidelberg: Springer; 2013: 151-160

[5] El Tecle NE, Abode-lyamah KO, Hitchon PW et al. Management of spinal fractures in patients with ankylosing spondylitis. Clin Neurol Neurosurg 2015; 139: 177-182

[6] Gallie E. Fractures and dislocations of the cervical spine. Am J Surg 1939; 46: 495-499

[7] Ghori A, Le HV, Makanji $\mathrm{H}$ et al. Posterior fixation techniques in the subaxial cervical spine. Cureus 2015; 7: e338

[8] Harms J, Melcher RP. Posterior C1-C2 fusion with polyaxial screw and rod fixation. Spine (Phila Pa 1976) 2001; 26: 24672471

[9] Jeanneret B, Gebhard JS, Magerl F. Transpedicular screw fixation of articular mass fracture-separation: results of an anatomical study and operative technique. J Spinal Disord 1994; 7: 222-229

[10] Judet R, Roy-Camille R, Saillant G. Actualités de chirurgie orthopédique de l'Hôpital Raymond-Poincaré: fracture du rachis cervical. Paris: Masson; 1970: 174-195

[11] Kantelhardt SR, Keric N, Conrad J et al. Minimally invasive instrumentation of uncomplicated cervical fractures. Eur Spine J 2016; 25: 127-133

[12] Matschke S, Wendl K, Gruetzner PA et al. Densfraktur mit begleitender traumatischer atlantoaxialer Instabilität. Trauma Berufskrankh 2016; 18: 289-295

[13] Matschke S, Krämer P, Wendl K et al. Verletzungen der subaxialen Halswirbelsäule. Aktuelle Therapiestandards. Trauma Berufskrankh 2015; 17 (Suppl. 2): S307-S314
[14] Oberkircher L, Born S, Struewer ] et al. Biomechanical evaluation of the impact of various facet joint lesions on the primary stability of anterior plate fixation in cervical dislocation injuries: a cadaver study. J Neurosurg Spine 2014; 21: 634-639

[15] Pingel A, Castein J, Kandziora F. Posterior stabilization of the cervical spine with lateral mass screws. Eur Spine J 2015; 24 (Suppl. 8): S947-S948

[16] Reindl R, Ouellet J, Harvey E] et al. Anterior reduction for cervical spine dislocation. Spine (Phila Pa 1976) 2006; 31: 648652

[17] Richter M, Cakir B, Schmidt R. Cervical pedicle screws: conventional versus computer-assisted placement of cannulated screws. Spine (Phila Pa 1976) 2005; 30: 2280-2287

[18] Schleicher P, Scholz M, Kandziora F et al. [Subaxial cervical spine injuries: treatment recommendations of the German Orthopedic and Trauma Society]. Z Orthop Unfall 2017; 155: 556-566. doi:10.1055/s-0043-110855

[19] Theologis AA, Burch S. Safety and efficacy of reconstruction of complex cervical spine pathology using pedicle screws inserted with stealth navigation and 3D image-guided (O-Arm) technology. Spine (Phila Pa 1976) 2015; 40: 1397-1406

[20] Tofuku K, Koga H, Yone K et al. Distractive flexion injuries of the subaxial cervical spine treated with a posterior procedure using cervical pedicle screws or acombined anterior and posterior procedure. J Clin Neurosci 2013; 20: 697-701

[21] Vaccaro AR, Koerner JD, Radcliff KE et al. AOSpine subaxial cervical spine injury classification system. Eur Spine | 2016; 25: 2173-2184

[22] Wendl K, Kreinest M, Matschke S et al. Dorsale Stabilisierungstechniken bei subaxialen Verletzungen der Halswirbelsäule. Trauma Berufskrankh 2016; 18: 289-295

[23] Wu JC, Huang WC, Chen YC et al.2008 Stabilization of subaxia cervical spines by lateral mass screw fixation with modified Magerl's technique. Surg Neurol 70: 25-33

Bibliografie

DOI https://doi.org/10.1055/a-0579-8992

OP-JOURNAL 2018; 34: 152-160 @ Georg Thieme Verlag KG Stuttgart · New York ISSN 0178-1715 